Check for updates

Cite this: Mater. Adv., 2021, 2, 2021

Received 23rd October 2020, Accepted 9th February 2021

DOI: 10.1039/d0ma00826e

rsc.li/materials-advances

\title{
Structure-controlled growth of vertically-aligned carbon nanotube forests using iron-nickel bimetallic catalysts $\dagger$
}

\author{
Deniz Ürk, (DD ${ }^{\text {ab }}$ Fevzi Çakmak Cebeci, ${ }^{\text {cd }}$ Mustafa Lütfi Öveçoğlu ${ }^{b}$ and \\ Hülya Cebeci (iD *ae
}

\begin{abstract}
Although the progress towards catalyst engineering and growth parameters to tune the properties of carbon nanotubes have been widely studied, critical properties like chirality still need addressing in the case of bimetallic catalysts. Herein, we synthesized six different catalysts via solid-state reactions using a mechanical alloying (MA) method to investigate the role that these catalysts have in the growth of highquality vertically aligned carbon nanotubes (VACNTs). The growth of the VACNTs was performed using a chemical vapor deposition method onto silicon wafers. Focusing on the impact of Ni addition into the Fe matrix, we explored the diameter distribution and chirality profiles of the VACNTs. The conductive band branches, the overall mapped morphology, chemical purity, structural quality, and physical alignment of the VACNTs were analysed in detail using Raman micro spectroscopy, thermogravimetric analysis, and scanning electron and transmission electron microscopies. The relative radial breathing modes were explored for the $(12,3),(9,3),(9,6)$, and $(10,4)$ chiralities, with different counts for overall samples. Furthermore, the $(11,9)$ chirality index, which is the most encountered type by far, was observed in the synthesis of VACNTs on the Fe $50 \mathrm{Ni}$ catalyst, with a relatively high count of $28 \%$. The current Fe-Ni bimetallic catalyst study thus employs Raman spectroscopy to determine the role that $\mathrm{Ni}$ addition into the Fe matrix has in the possible difference in the conductive branches of the VACNTs.
\end{abstract}

\section{Introduction}

Vertically aligned carbon nanotubes (VACNTs) have received much attention in the few last decades due to their use in many applications, including polymer nanocomposites (PNCs), ${ }^{1,2}$ nanoporous membranes, ${ }^{3,4}$ supercapacitor electrodes ${ }^{5,6}$ etc. However, the use of carbon nanotubes (CNTs) in demanding applications such as electronics, supercapacitors and/or sensors is still limited due to their low quality in batch production with a narrow diameter and non-selective chirality growth. ${ }^{7}$ Even though VACNTs can be easily synthesized using monometallic transition metals such as $\mathrm{Fe}, \mathrm{Ni}, \mathrm{Co}, \mathrm{Mo}, \mathrm{Cu}$ etc., which have

\footnotetext{
${ }^{a}$ Aerospace Research Center, Istanbul Technical University, Istanbul 34469, Turkey. E-mail: hulya.cebeci@itu.edu.tr

${ }^{b}$ Department of Metallurgical and Materials Engineering, Istanbul Technical University, Istanbul 34469, Turkey

${ }^{c}$ Faculty of Engineering and Natural Sciences, Sabanci University, Istanbul 34956, Turkey

${ }^{d}$ Sabanci University SUNUM Nanotechnology Research Center, 34956, Turkey

${ }^{e}$ Faculty of Aeronautics and Astronautics, Istanbul Technical University, Istanbul 34467, Turkey

$\dagger$ Electronic supplementary information (ESI) available. See DOI: 10.1039/ d0ma00826e
}

unique properties related to their $3 \mathrm{~d}$-orbitals, it is still a challenge to synthesize fully aligned CNTs with few defects at a high density that are homogeneous and show controlled chirality specifically for electronic applications. ${ }^{8-12}$ Recent studies have been reported that bimetallic catalysts have the potential for size control, quality enhancement and chirality distribution compared to monometallic transition metals since these mixed metal catalysts have a combined effect on the overall properties besides characterized by complex relations such as catalyst particle size and combination. ${ }^{13-16}$

To date, controlling the diameter of CNTs with various methods has been proposed through changing the catalyst particle types (monometallic or bimetallic), modifying the carbon source $\left(\mathrm{CH}_{4}, \mathrm{C}_{2} \mathrm{H}_{2}, \mathrm{C}_{2} \mathrm{H}_{4}\right.$, etc. $)$, varying the feedstock flux, support materials (e.g. $\mathrm{SiO}_{2}, \mathrm{Al}_{2} \mathrm{O}_{3}, \mathrm{MgO}$, etc.), surface treatments (e.g. chemical etching, ion bombardment, etc.) reaction time and growth temperature. ${ }^{17-19}$ However, with a large number of variables, it is still a hurdle to achieve control over the tube diameter, which has a direct influence on the chiral distribution of the material. ${ }^{20}$ Recently, the most common and highest yielding growth method for VACNTs has been reported to be catalytic chemical vapor deposition (CCVD), which can be used to synthesize CNTs via a well-accepted 
vapor-liquid-solid (VLS) growth mechanism. ${ }^{21,22}$ According to the VLS mechanism, the catalyst nanoparticles can be viewed as a seed for the nucleation and growth of CNTs, where the diameter, areal density and number of walls firmly depend on the size of the catalyst nanoparticles. ${ }^{23,24}$ However, Liu et al. reported that as a monometallic catalyst, Fe nanoparticles tend to diffuse into the substrate at a high reaction temperature, which limits the achievement of a uniform diameter distribution of the VACNTs despite its high catalytic efficiency. ${ }^{25}$ Additionally, many researchers have also reported that bimetallic catalyst nanoparticle particles such as $\mathrm{FeMo},{ }^{26} \mathrm{FeNi}^{27-29}$ and $\mathrm{FeCu}^{30}$ improve the reducibility of $\mathrm{Fe}$ and inhibit the aggregation of $\mathrm{Fe}$ clusters, different from monometallic catalysts. Li et al. achieved controlled diameter distribution, wall number and thermal stability of VACNTs grown on a FePt catalyst compared to VACNTs grown from an Fe catalyst. ${ }^{31}$

Aside from diameter control, quality enhancement is another critical issue for CNTs for a wide range of applications. ${ }^{32}$ Apart from the mobility of the carbon source increasing with temperature, the CNT growth rate is also proportional to the reaction temperature owing to the high face-centered cubic phase of $\mathrm{Fe}\left(\gamma_{\mathrm{Fe}}\right) \cdot{ }^{33}$ Lee et al. determined that both lateral alignment and crystallinity of CNTs are extremely dependent on the growth temperature using Raman spectroscopy. ${ }^{34}$ However, when the optimal temperature is reached, the CNT growth rate decreases due to the accumulation of amorphous carbon on the catalyst surface and deactivates the catalyst particles which reduce the CNT quality in the presence of the amorphous carbon. ${ }^{35,36}$ Hence, Hata et al. developed a new technique for the super growth of CNTs and they significantly increased the catalytic activity by removing amorphous carbon through the addition of water vapor. ${ }^{37}$ Moreover, Amama et al. reported that water vapor hinders the diffusion and Ostwald ripening of catalyst particles through the mitigation of oxygen and hydroxyl groups. ${ }^{38}$ Consequently, several researchers focused on $\mathrm{Co}-\mathrm{Cu}$, Fe-Mo, Fe-Ni bimetallic catalyst particles to inhibit Ostwald ripening and reported that the synthesis of CNTs with low defect levels and enhanced crystallinity was achieved. ${ }^{26,32,39}$

The main bottleneck in the development of CNT-based technologies such as electronics and optics is the precise control of the chirality of CNTs that have high-crystallinity, a low-defect density and low diameter. ${ }^{40}$ As such, huge efforts have been dedicated towards chirality controlled synthesis in the last few years, including optimizing growth conditions such as the growth temperature, the carbon source type, support materials, surface treatments and the design of the catalyst nanoparticles. ${ }^{41-43}$ Many researchers have focused on understanding how the catalyst composition and the structure affect the chirality distribution of CNTs. ${ }^{44}$ Despite several studies being reported, establishing a link between the catalyst properties and CNT structures still remains elusive owing to the complex relationship between them. ${ }^{45,46}$ Thus, density functional theory (DFT) calculations have led to the investigation of the epitaxial relationships between bimetallic catalyst nanoparticles such as $\mathrm{Ni}_{x} \mathrm{Fe}_{1-x}, \mathrm{FeGa}$, NiGa, and Pd-Co and nanotube cap formation. ${ }^{47-49}$ However, DFT calculations are carried out at zero temperature, so they only provide a thermodynamic overview of nanotube nucleation and growth. ${ }^{49}$ Rao et al. determined that kinetic aspects are important in assigning the chirality of CNTs using in situ Raman spectroscopy. ${ }^{50}$ Thus, experimental studies are important for determining the selective-chirality of CNTs combined with molecular dynamics simulations. ${ }^{39}$

In this study, through the use of six distinct catalyst types based on Fe-Ni combinations with different weight fractions, VACNT synthesis via CCVD was investigated using Raman spectroscopy, thermogravimetric analysis (TGA), scanning electron microscopy (SEM) and transmission electron microscopy (TEM). The main goal of this study is to determine the effects that catalyst composition have on several parameters, including the chirality index, diameter, quality, purity, and morphology of the VACNTs. Fe-Ni based catalysts with different weight fractions were synthesized using a mechanical alloying (MA) method. These catalysts were then used to coat silicon (Si) wafers using the E-beam method. The CCVD parameters were kept the same for all of the catalyst types used in this study to compare the effect of the catalysts. Subsequently, the synthesized VACNTs were characterized by Raman spectroscopy. A Lorentzian area function was utilized to fit the peaks of the radial breathing mode (RBM) for each sample and chirality indexes of $(n, m)$ were assigned from Kataura plots. Furthermore, the morphological and oxidation behavior of the CNTs were evaluated using SEM, TEM and TGA. We revealed that the counts of the special chirality species such as $(11,9),(9,3),(9,6)(12,3)$ and $(10,4)$ significantly change upon the addition of Ni into the Fe matrix. In this way, this study paves the way for the selective growth of VACNTs depending on their chiral index in future studies.

\section{Experimental study}

\subsection{Preparation of the bimetallic catalysts}

MA was performed to prepare Fe-Ni powder alloy catalysts from high purity powders (Fe $99.9 \%$ purity, Ni $99.5 \%$ from the ABCR Corporation) through varying the $\mathrm{Ni}$ amounts in the catalysts to $5,10,15,20$ and $50 \mathrm{wt} \%$. Powder blends were milled for $8 \mathrm{~h}$ using a Spex $8000 \mathrm{D}$ ball mill at a rotational speed of $1200 \mathrm{rpm}$. A ball-to-powder weight ratio (BPR) of 10:1 using stainless steel balls (diameter $6 \mathrm{~mm}$ ) in a stainless-steel box was employed for MA. Milling vials were placed into a glove box (PlasmaLab TM) under an Ar atmosphere (Linde TM 99.999\% purity) to protect oxidation of the powders at high speed. All of the powder alloy catalysts were annealed at $600{ }^{\circ} \mathrm{C}$ for $2 \mathrm{~h}$ to remove the residual stress that arises from the MA process.

\subsection{Synthesis of the VACNTs by CCVD}

VACNTs were synthesized using the CCVD method at $750{ }^{\circ} \mathrm{C}$ on highly n-doped $\mathrm{Si}$ wafer substrates coated with $\mathrm{Al}_{2} \mathrm{O}_{3}$ /catalyst using an E-beam. The Si substrate was first coated with a $300 \mathrm{~nm}$ oxide layer via plasma-enhanced chemical vapor deposition (Oxford Instrument Plasma Lab System 100) and then E-beam evaporation was performed to obtain a uniform $\mathrm{Al}_{2} \mathrm{O}_{3}$ thin film of $10 \mathrm{~nm}$ in thickness as a buffer layer at a rate 
(a)

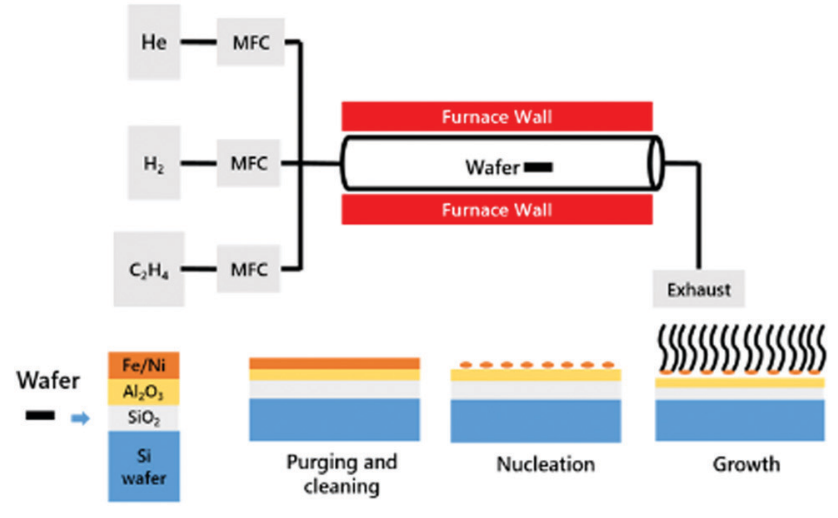

(b)
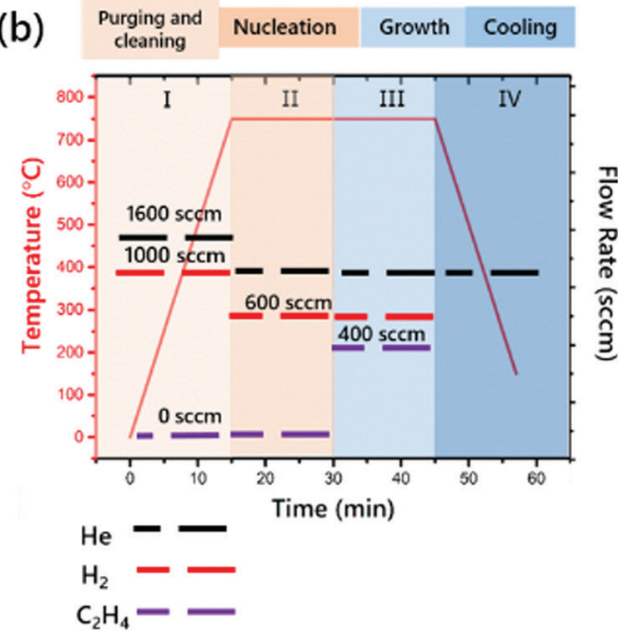

Fig. 1 (a) Schematic of the CCVD system and growth mechanism of VACNTs onto Si wafers and (b) the growth protocol for the preparation of VACNTs in a quartz tube furnace using $\mathrm{He}, \mathrm{H}_{2}$ and $\mathrm{C}_{2} \mathrm{H}_{4}$ as gas sources.

of $1 \AA \mathrm{s}^{-1}$ and a synthesized binary catalyst with a thickness of $2 \mathrm{~nm}$ at $2-410^{-6}$ torr (Torr Instrument) at a rate of $0.1 \AA \mathrm{s}^{-1}$. A single zone quartz tube furnace was used for the CCVD process (Lindberg/Blue M) equipped with an electronic mass flow controller (Alicat Inc.) (Fig. 1(a)). CNT growth was performed under atmospheric pressure using the reactant gases $\mathrm{C}_{2} \mathrm{H}_{4}$, $\mathrm{H}_{2}$ and He. The Si-wafer samples were placed into the hot zone of the furnace, as shown in Fig. 1(a). After purging and cleaning of the quartz tubes using $\mathrm{He}$ and $\mathrm{H}_{2}$, the furnace was heated to $750{ }^{\circ} \mathrm{C}$ with $1600 \mathrm{sccm} \mathrm{He}$ and $1000 \mathrm{sccm} \mathrm{H}_{2}$ for $15 \mathrm{~min}$ to nucleate the catalyst particles. Then, $1000 \mathrm{sccm} \mathrm{He}, 600 \mathrm{sccm} \mathrm{H}_{2}$ and $400 \mathrm{sccm} \mathrm{C}_{2} \mathrm{H}_{4}$ were introduced into the quartz tubes for $15 \mathrm{~min}$. The aforementioned four main steps are summarized in Fig. 1(b), showing the growth conditions such as temperature, time and gas flow rate.

\subsection{Characterization of the VACNTs}

VACNTs were characterized using SEM (FEI QUANTA) with an acceleration voltage in the range of $5-15 \mathrm{kV}$ under high vacuum. Also, TEM analysis was performed using a JEOL ARM-200 CFEG microscope operated at $200 \mathrm{kV}$ equipped with a Cs probe collector (JEOL, Tokyo) to measure the tube diameter and number of tube walls in the VACNTs. To prepare the TEM samples, VACNTs were immersed in isopropyl alcohol and ultrasonicated for $5 \mathrm{~h}$. Then, a drop of sample was placed on a carbon coated $300 \mu \mathrm{m}$ mesh size $\mathrm{Cu}$ grid. The grid was dried at room temperature and cleaned using plasma for 10 seconds before loading on the microscope. Raman spectroscopy was performed using a Renishaw inVia reflex microscope and spectrometer with an excitation energy of $2.33 \mathrm{eV}$ over an acquisition range of $100-3200 \mathrm{~cm}^{-1}$, coupled with an ultraviolet (UV) and near-infrared (NIR) enhanced deep depletion, Peltier cooled CCD array detector $(1024 \times 256$ pixels $)$ and a Leica microscope equipped with $5 \times, 20 \times$ and $50 \times$ magnification objectives. A computer controlled $X Y Z$ stage with a scan area of $40 \times 40 \mathrm{~mm}$ $(X$ and $Y$ ) and a step size of $1 \mathrm{~mm}$ allowed automated Raman spectral mapping measurements (laser power $\sim 1 \mathrm{~mW}$ in $1 \mu \mathrm{m}^{2}$ ) to be carried out. TGA was performed using an SDT Q600 TGA thermogravimetric analyzer at a heating rate of $10{ }^{\circ} \mathrm{C} \mathrm{min}{ }^{-1}$ up to $800{ }^{\circ} \mathrm{C}$ under a $100 \mathrm{ml} \mathrm{min}{ }^{-1}$ airflow.

\section{Results and discussion}

\subsection{Characterization of the Fe-Ni catalysts}

The phase components, crystal structures and directions of the planes were detected by X-ray diffractometry (XRD) using a Bruker $^{\mathrm{TM}} \mathrm{D} 8$ advance diffractometer (CuK $\alpha$ radiation, $\lambda=1.542 \AA$ ). As shown in Fig. S1 (ESI $\dagger$ ), the peaks marked with , at $44.8^{\circ}, 66.1^{\circ}$, and $82.4^{\circ}$ can be indexed to the (110), (200) and (211) planes of BCC Fe-Ni. A small peak marked with $\bullet$ at $51.1^{\circ}$ attributed to the (200) FCC plane of the Fe-Ni alloy was present in the XRD pattern of Fe20Ni. Ni is a well-known austenite phase stabilizer used in the steel industry. ${ }^{51} \mathrm{~A}$ detailed investigation showed that the presence of $\mathrm{Ni}$ in an amount higher than $18.4 \mathrm{wt} \%$ stabilized the FCC-Fe content of the material into a BCC-Fe structure after the annealing process. ${ }^{52}$ Moreover, increasing the Ni content results in an increase in the stabilized FCC-Fe content. The XRD pattern of Fe50Ni consists of diffraction peaks marked with $*$ at $43.8^{\circ}, \bullet$ at $51.1^{\circ}$ and ${ }^{*}$ at $75.2^{\circ}$, which can be attributed to an Fe0.5Ni0.5 alloy with a FCC crystalline structure. ${ }^{53}$ The main diffraction peak of the FCC Fe-Ni alloy at the $2 \theta$ position of $43.8^{\circ}$, which cannot be detected in the XRD pattern of $\mathrm{Fe} 20 \mathrm{Ni}$, may be caused by overlapping with the dominant peak of the BCC-Fe structure at $44.8^{\circ}$. Moreover, there was no trace of the peaks belonging to the BCC-Fe crystalline phase in the XRD pattern of Fe50Ni, which can be attributed to the existence of the Fe50Ni catalyst only in a FCC structure. In addition to that, when the $\mathrm{Fe}-\mathrm{C}$ phase diagram was considered, the carbon solubility of the FCC structure was found to be higher than that of the BCC structure. Hence, by increasing the amount of $\mathrm{Ni}$ in Fe-Ni alloy, the carbon solubility in the FCC structure improves. ${ }^{12}$ 
3.2 The effects of Ni addition on the chirality and diameter of the VACNTs

Identifying the effects that the Ni addition in the catalyst alloy has on the chirality and diameter of the VACNTs was studied by Raman spectroscopy from the RBM peaks between 120 and $300 \mathrm{~cm}^{-1}$. Using an empirical Kataura plot, ${ }^{55}$ these RBM peaks were correlated with the chirality of the VACNTs. The diameter of the VACNTs was calculated as $\omega_{\mathrm{RBM}}=227 / d_{t},{ }^{39}$ where $\omega$ is the Raman shift of the RBM peaks at 121 different points and the RBM spectra of the VACNTs were fitted using a Lorentzian area function. For each fitting, the $R$-squares adj. values were higher than 0.95 . The positions of the RBM peaks are listed in Table 1 according to their RBM peak positions, assignment of the chirality, diameters, and type of conductivity, respectively (see more detailed information in Table S1 in the ESI, $\dagger$ which also gives more information about the equations).

Fig. 2 presents the RBM region of the spectrum at $2.33 \mathrm{eV}$ with metallic or semiconducting branches. For quantitative evaluation of each peak depending on the diameter and chirality, the integrated intensities of the RBMs were employed in the Raman spectra. ${ }^{58,59}$

Table 1 RBM shifts of the synthesized VACNTs according to their Fe and Fe-Ni alloy combinations for green laser spots $(2.33 \mathrm{eV}, 532 \mathrm{~nm})$ used in all the calculations ( $\omega_{\mathrm{RBM}}$ : mean value from 121 spectra $)^{40,56,57}$

\begin{tabular}{|c|c|c|c|c|}
\hline Catalyst type & $\omega_{\mathrm{RBM}}\left(\mathrm{cm}^{-1}\right)$ & $(n, m)$ & $d(\mathrm{~nm})$ & $\mathrm{S}$ or $\mathrm{M}$ \\
\hline \multirow[t]{6}{*}{ Fe-Only } & 165 & $(11,9)$ & 1.37 & S \\
\hline & 187 & $(12,5)$ & 1.20 & $\mathrm{~S}$ \\
\hline & 208 & $(12,3)$ & 1.09 & M \\
\hline & 220 & $(9,6)$ & 1.03 & $\mathbf{M}$ \\
\hline & 229 & $(10,4)$ & 0.99 & $\mathbf{M}$ \\
\hline & 267 & $(9,3)$ & 0.85 & M \\
\hline \multirow[t]{7}{*}{$\mathrm{Fe} 5 \mathrm{Ni}$} & 131 & $(15,10)$ & 1.73 & $\mathrm{~S}$ \\
\hline & 162 & $(16,3)$ & 1.40 & S \\
\hline & 182 & $(10,8)$ & 1.24 & S \\
\hline & 208 & $(12,3)$ & 1.09 & M \\
\hline & 220 & $(9,6)$ & 1.03 & M \\
\hline & 229 & $(10,4)$ & 0.99 & $\mathbf{M}$ \\
\hline & 267 & $(9,3)$ & 0.85 & M \\
\hline \multirow[t]{7}{*}{ Fe10Ni } & 135 & $(16,8)$ & 1.68 & S \\
\hline & 164 & $(11,9)$ & 1.37 & $\mathrm{~S}$ \\
\hline & 185 & $(15,1)$ & 1.23 & $\mathrm{~S}$ \\
\hline & 208 & $(12,3)$ & 1.09 & M \\
\hline & 220 & $(9,6)$ & 1.03 & $\mathbf{M}$ \\
\hline & 230 & $(10,4)$ & 0.99 & $\mathbf{M}$ \\
\hline & 266 & $(9,3)$ & 0.85 & $\mathbf{M}$ \\
\hline \multirow[t]{6}{*}{$\mathrm{Fe} 15 \mathrm{Ni}$} & 162 & $(16,3)$ & 1.40 & $\mathrm{~S}$ \\
\hline & 187 & $(12,5)$ & 1.20 & $\mathrm{~S}$ \\
\hline & 208 & $(12,3)$ & 1.09 & M \\
\hline & 220 & $(9,6)$ & 1.03 & $\mathbf{M}$ \\
\hline & 229 & $(10,4)$ & 0.99 & $\mathbf{M}$ \\
\hline & 267 & $(9,3)$ & 0.85 & M \\
\hline \multirow[t]{7}{*}{$\mathrm{Fe} 20 \mathrm{Ni}$} & 157 & $(11,10)$ & 1.44 & $\mathrm{~S}$ \\
\hline & 188 & $(12,5)$ & 1.20 & S \\
\hline & 210 & $(12,3)$ & 1.09 & M \\
\hline & 221 & $(9,6)$ & 1.03 & M \\
\hline & 231 & $(10,4)$ & 0.98 & $\mathbf{M}$ \\
\hline & 267 & $(9,3)$ & 0.85 & M \\
\hline & 286 & $(10,0)$ & 0.79 & $\mathrm{~S}$ \\
\hline \multirow[t]{4}{*}{ Fe50Ni } & 165 & $(11,9)$ & 1.37 & $\mathrm{~S}$ \\
\hline & 220 & $(9,6)$ & 1.03 & M \\
\hline & 229 & $(10,4)$ & 0.98 & M \\
\hline & 267 & $(9,3)$ & 0.85 & M \\
\hline
\end{tabular}

As clearly presented, the diameter distribution is wide, ranging from 0.79 to $1.73 \mathrm{~nm}$, and the most common species are at $1.20,1.09,1.03$ and $0.85 \mathrm{~nm}$ for the six samples. The mean diameters of the CNTs are close and in between 1.01 and $1.20 \mathrm{~nm}$. The RBM peaks were observed at 131 and $135 \mathrm{~cm}^{-1}$ for the VACNTs synthesized using Fe5Ni and Fe10Ni, respectively. These peaks were presented from the $S_{44}$ branch in the Kataura plot and they were not seen in other samples. However, as the branches $S_{33}$ and $S_{44}$ overlap at $\lambda_{\text {laser }}=532 \mathrm{~nm}$, utilizing a higher laser power is more convenient to detect larger tube diameters, as also indicated in the enlarged Kataura plot. ${ }^{60}$ An RBM peak was observed at $165 \mathrm{~cm}^{-1}$ for VACNTs synthesized using Pure-Fe, Fe10Ni, and Fe50Ni. Fig. 2(a), (b) and (f) reveal that these VACNTs feature $(11,9)$ as the most abundant chiral species, with concentrations of $14 \%, 14 \%$ and $28 \%$, respectively.

This value was calculated as approximately $17 \%$ for the $(11,9)$ chirality in previous reports. ${ }^{58}$ Additionally, for the Pure-Fe, Fe15Ni, and Fe50Ni catalysts, the VACNTs presented a peak at $187 \mathrm{~cm}^{-1}$. In the $S_{33}$ branch, only this peak was detected for VACNTs synthesized using Fe50Ni. However, the most intense peaks were observed at 208, 220, 229 and $267 \mathrm{~cm}^{-1}$ for all samples in the $\mathbf{M}_{11}$ branch. Cheng et al. reported the same RBM peaks for bundled HiPco SWCNTs at an excitation energy of $2.33 \mathrm{eV} .^{61}$ According to the RBM in the spectrum of the VACNTs synthesized using Fe10Ni, it was clearly seen that semiconducting VACNTs in 55\% were grown compared to the other samples, meaning that they could have important potential use as high-performance field effect transistors after enrichment processes. ${ }^{62-64}$

Exploring the structural quality of the large-scale VACNTs, Raman spectral mappings were recorded for all of the samples, on $40 \times 40 \mu \mathrm{m}^{2}$ scan areas, with a step size of $5 \mu \mathrm{m}$. Raman mappings of VACNTs synthesized on Pure-Fe, Fe5Ni, Fe10Ni, Fe15Ni, Fe20Ni, and Fe50Ni are presented in Fig. 3. The G-band, observed at $1600 \mathrm{~cm}^{-1}$, is the first-order Raman scattering band, related to the degree of graphitization, while the defect-induced D-band provides information about the side walls of the CNTs. ${ }^{65,66}$ The quality of a sample is often identified from the intensity of the G- to D-band ratio. ${ }^{67}$ The mappings of VACNTs synthesized on different catalyst particles are shown in Fig. 3, using different colors for each $I_{\mathrm{G}} / I_{\mathrm{D}}$ ratio. The means of the $I_{\mathrm{G}} / I_{\mathrm{D}}$ ratios of VACNTs synthesized on Pure-Fe, Fe5Ni, Fe10Ni, Fe15Ni, Fe20Ni, and Fe50Ni are approximately 2.04, 2.09, 2.68, 2.07, 1.75 and 1.73 , respectively. The maximum $I_{\mathrm{G}} / I_{\mathrm{D}}$ ratio was obtained for the VACNTs synthesized on Fe10Ni. Although this value is higher than the others, it does not give information on the types of the defects in the sample because perfect zigzag edges, charge impurities, intercalants, uniaxial and biaxial strain do not generate a D-band peak. ${ }^{68}$

Cancado et al. calculated the defect density in a graphene structure using Raman spectroscopy, employing different laser powers. ${ }^{68}$ They presented the relationships between $I_{\mathrm{D}} / I_{\mathrm{G}}$ and the distance between the defects $\left(L_{\mathrm{D}}\right)$ depending on the excitation laser wavelength $\lambda_{\mathrm{L}}(\mathrm{nm})$, using the simple equation shown in eqn (1). They also reported that this empirical relationship can be 

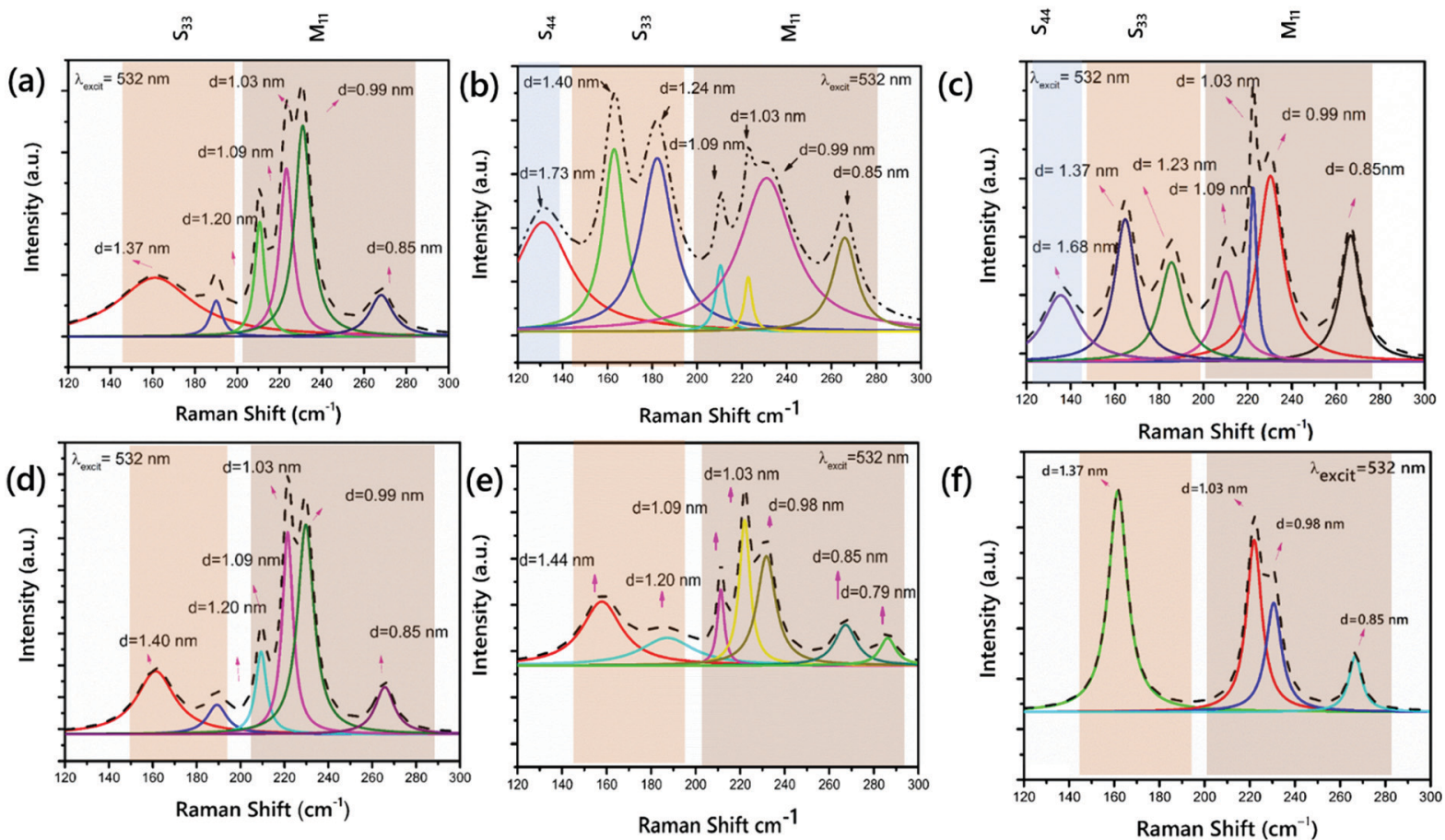

Fig. 2 RBM peaks fitted to a Lorentzian area function and calculation of the innermost diameters of the VACNTs synthesized on (a) Pure-Fe nanoparticles, (b) Fe5Ni, (c) Fe10Ni, (d) Fe15Ni, (e) Fe20Ni, and (f) Fe50Ni ( $E_{\text {Laser }}=2.33$. eV).

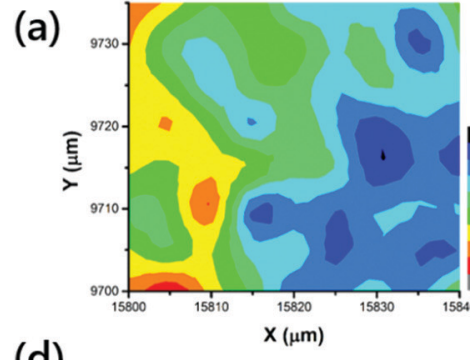

(d)

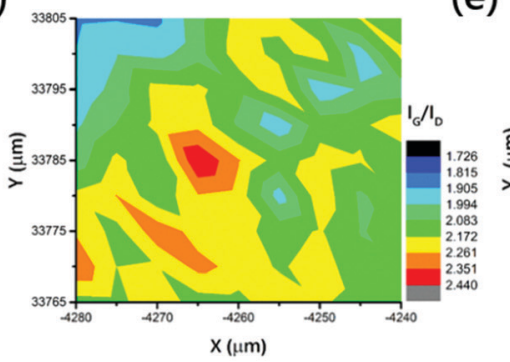

(b)

(e)
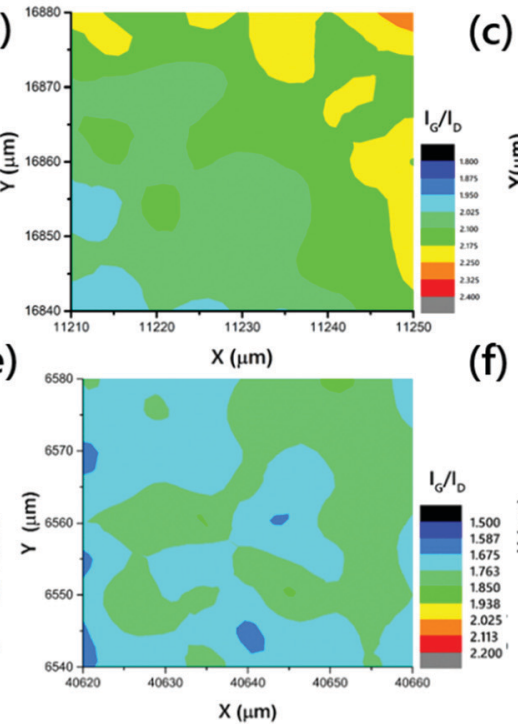

(c)

(f)
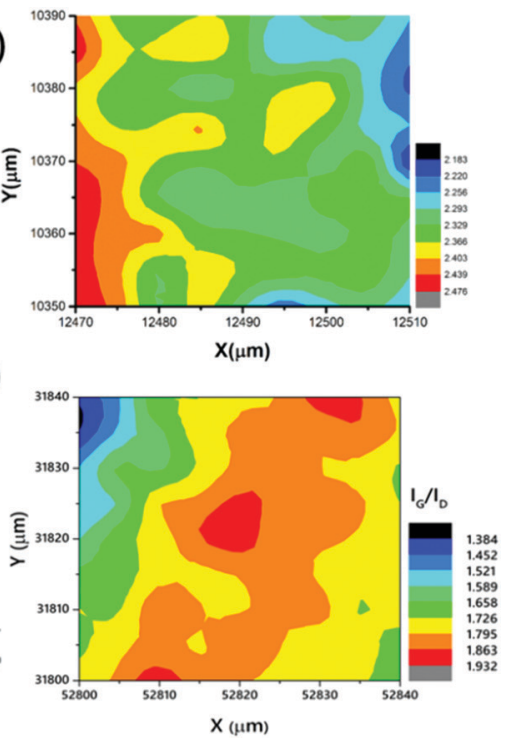

Fig. 3 Raman mappings of the VACNTs synthesized on (a) Pure-Fe, (b) Fe5Ni, (c) Fe10Ni, (d) Fe15Ni, (e) Fe20Ni, and (f) Fe50Ni.

used for $L_{\mathrm{D}}>10 \mathrm{~nm}$ in Raman spectroscopy for any laser line in the visible range. ${ }^{68}$

$$
L_{\mathrm{D}}^{2}\left(\mathrm{~nm}^{2}\right)=(1.8 \mp 0.5) \times 10^{-9} \lambda_{\mathrm{L}}^{4}\left(\frac{I_{\mathrm{D}}}{I_{\mathrm{G}}}\right)^{-1}
$$

According to the calculations of $L_{\mathrm{D}}$ for all of the samples, the defect density of the VACNTs synthesized in this study using Fe10Ni is lower than other catalyst combinations as the distance between the defects is greater, as presented in Fig. 4. In this calculation, 121 different $I_{\mathrm{G}} / I_{\mathrm{D}}$ ratios were input into eqn (1). that correspond to Raman active defects. ${ }^{68}$ It is critical to note here that silent defects such as perfect zigzags, intercalants, and impurities were not considered as they are correlated to G-band and $2 \mathrm{D}$ peaks. ${ }^{69,70}$

\subsection{Defining the chiral indexes of VACNTs with various catalysts}

There are two main strategies for evaluating the chirality index $(n, m)$ of CNTs quantitatively. First, the counts for each chiral 


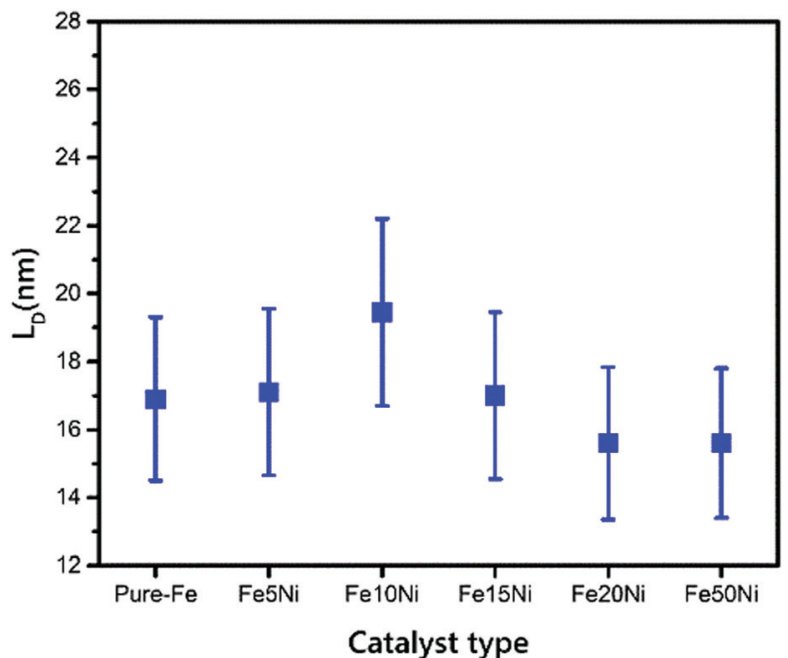

Fig. 4 Distance between defects with error bars for VACNTs synthesized on different catalyst particles.

index in a sample are assumed to be proportional to their integral RBM intensities. ${ }^{58}$ This aspect is commonly used for bulk samples (e.g. thin films, arrays, forests etc. ${ }^{63,71,72}$ ). The second strategy is to count the numbers of RBM peaks in the Raman spectral mapping. ${ }^{73}$ In this study, the ratios of RBM intensities for all RBM peaks were compared to each other. The root of the chirality index can be calculated from the diameter $\left(d_{\mathrm{t}}\right)$, found using $d_{t}=\sqrt{ } 3 a_{\mathrm{c}-\mathrm{c}} / \pi \sqrt{n^{2}+n m+m^{2}}$, where $a_{\mathrm{c}-\mathrm{c}}$ denotes the carbon-carbon bond length (as $0.144 \mathrm{~nm}$ ). According to the results represented in Fig. S3-S8 (ESI $\dagger$ ), in detail, all common tubes clearly fit the $\mathrm{M}_{11}$ branch. The effect of the catalyst particles on the chirality of the VACNTs, in terms of the relative RBM intensities, was evaluated for the $(9,6),(9,3),(12,3)$ and $(10,4)$ chiralities, which are plotted against the catalyst type in Fig. 5. Cheng et al. and Kharlamova et al. identified these same chiral vectors from RBM frequencies for their samples. ${ }^{61,74}$ It should be

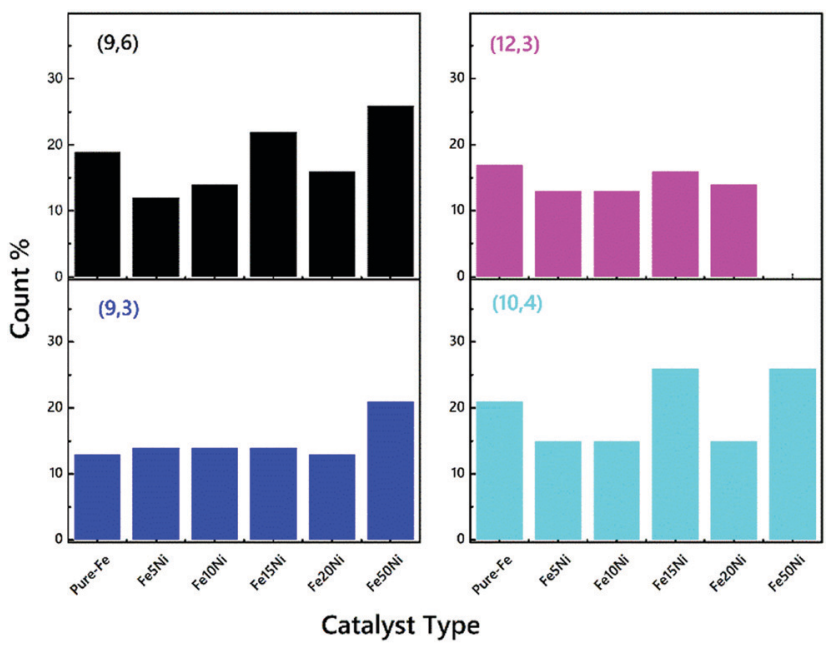

Fig. 5 The ratios of the CNTs with common $(9,6),(9,3),(12,3)$ and $(10,4)$ chiralities of the VACNTs synthesized using various catalyst particles. noted that although the correlation between catalyst particles and chirality distribution is reported in this study, a more detailed investigation using sophisticated instrumentation such as microscopic and spectroscopic techniques is necessary for achieving the exact values for using the in situ CCVD system.

\subsection{The effects of $\mathrm{Ni}$ addition on the oxidation behavior of the VACNTS}

To understand the decomposition and purity of VACNTs, TGA was carried out at $800{ }^{\circ} \mathrm{C}$ under a $100 \mathrm{ml} \mathrm{min}^{-1}$ airflow for all of the samples. According to the TGA results shown in Fig. S10 (ESI $\dagger$ ), the highest temperature of the maximum rate of the oxidation resulted in the growth of the VACNTs on the Fe5Ni catalyst alloy. According to the TGA results, the mass of the residual metal catalyst nanoparticles obtained was $\sim 2-3 \mathrm{wt} \%$ for each sample. The results of comparing the temperature of the maximum rate of oxidation depending on the catalyst type are shown in Table 2. Furthermore, these results can be correlated with the structural stability of the CNTs. In addition, the XRD pattern of the VACNTs is presented in Fig. S2 (ESI $\dagger$ ). The three characteristic diffraction peaks of the VACNTs with $2 \theta$ angles of around $25^{\circ}, 43^{\circ}$, and $77^{\circ}$ are associated with the $\mathrm{C}(002)$, C (100) and C (110) diffractions of graphite, respectively. ${ }^{54}$

\subsection{Physical properties of the VACNTs}

Fig. 6 shows micrographs of the VACNTs synthesized using neat $\mathrm{Fe}$ and $\mathrm{Fe}-\mathrm{Ni}$ alloy catalysts from 5 to $50 \mathrm{wt} \%$ of $\mathrm{Ni}$ addition through imaging using SEM at an acceleration voltage of $5-15 \mathrm{kV}$ at $50000 \times$ magnification. The growth mechanism was determined as base-mode. This is due to the strong interaction between the catalyst and surface (the top-view SEM image of the VACNTs is shown in Fig. S9, ESI $\dagger$ ). All of the samples show vertically aligned CNTs, without any significant difference in terms of their alignment. However, as depicted in Fig. 6(b), when the Fe5Ni alloy catalyst is used in the synthesis, an evident change was observed, with enhanced waviness and entanglements in between neighboring VACNTs. The carbon atoms precipitated during the catalyst cluster formation may present such entanglement and increased waviness similar to that observed in Fig. 6(b). According to ImageJ processing, the inter-wiring CNT-CNT spacing is approximately 75-80 nm for the synthesized VACNTs on both Pure-Fe and Fe5Ni. This is proof that the increase in waviness is due to the catalysis dynamics. This situation may have attributed to the high surface energy for the precipitation of carbon atoms. Li et al. conducted finite element

Table 2 TGA results of VACNTs synthesized using different catalyst nanoparticles

\begin{tabular}{ll} 
Type of catalyst & $\begin{array}{l}\text { Temperature of the maximum } \\
\text { rate of oxidation }\left({ }^{\circ} \mathrm{C}\right)\end{array}$ \\
\hline Pure-Fe & 696 \\
Fe5Ni & 704 \\
Fe10Ni & 700 \\
Fe15Ni & 651 \\
Fe20Ni & 668 \\
Fe50Ni & 703
\end{tabular}




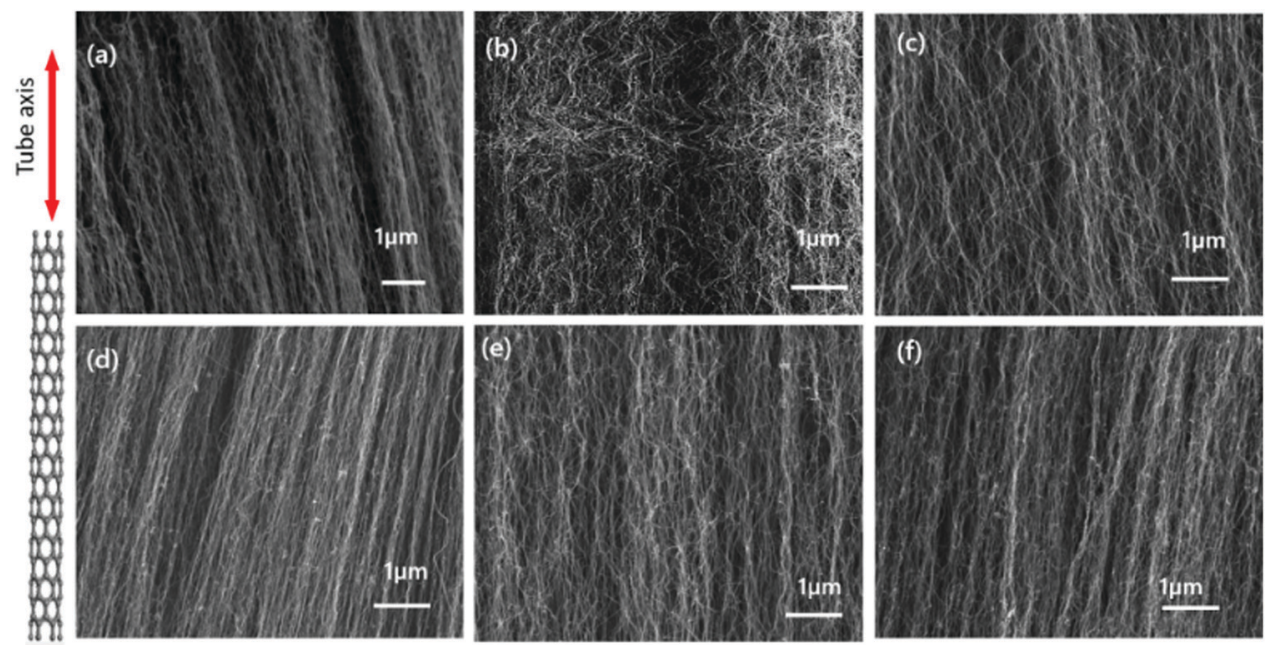

Fig. 6 FEG-SEM images of VACNTs synthesized on (a) Pure-Fe, (b) Fe5Ni, (c) Fe10Ni, (d) Fe15Ni, (e) Fe20Ni, and (f) Fe50Ni (scale bars: 1 rm).

simulations for CNT/polymer composites when CNT waviness was considered. The improved micromechanics model showed that
CNT waviness reduces the elastic modulus but enhances the ultimate strain of the composite. ${ }^{75}$ From morphological analysis,
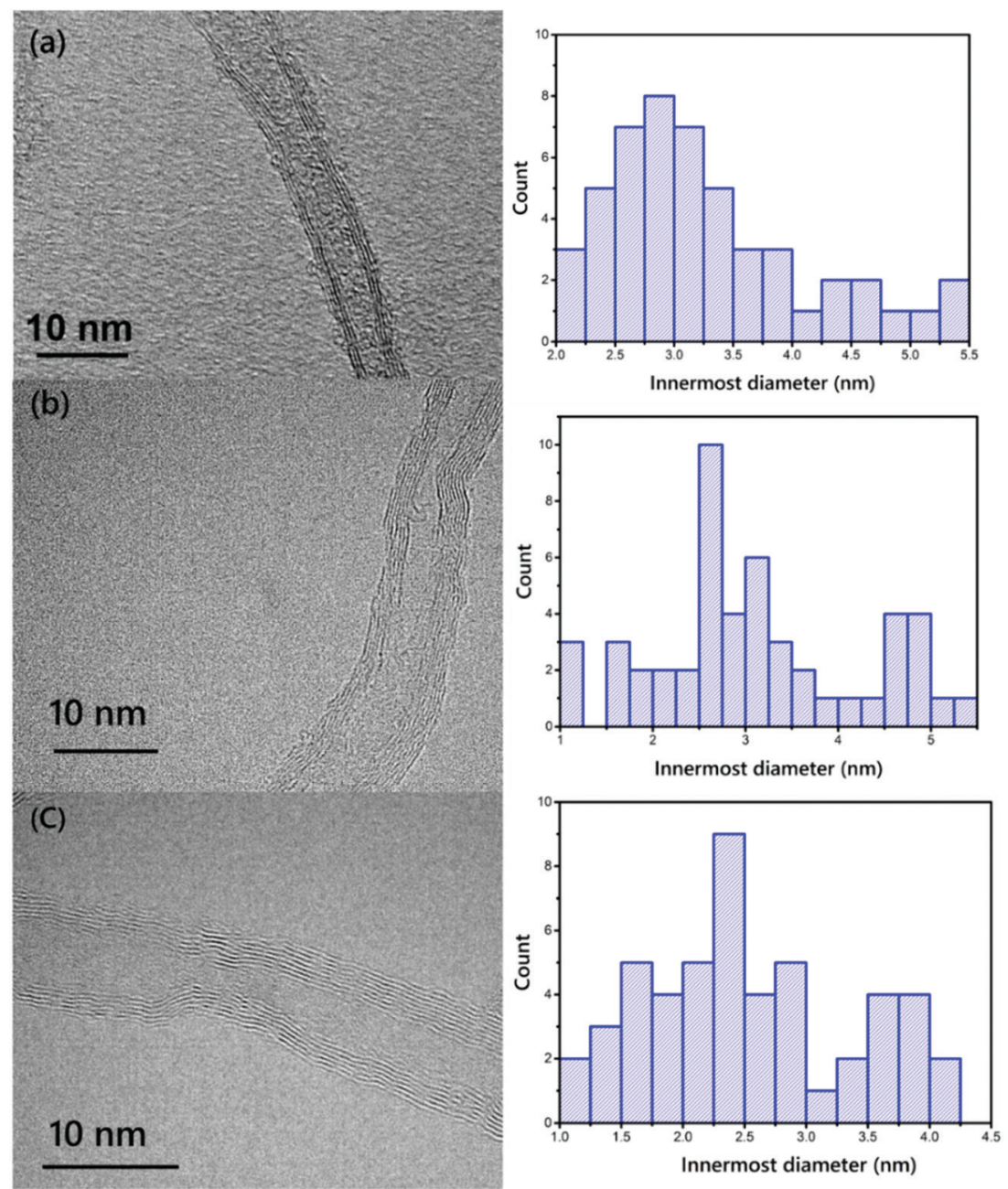

Fig. 7 TEM images and histograms of the distributions of the innermost diameters of the VACNTs synthesized on (a) Pure-Fe, (b) Fe5Ni, and (c) Fe50Ni (scale bars: $10 \mathrm{~nm}$ ). 
it can also clearly be concluded that the VACNTs synthesized using Fe5Ni have better potential as a reinforcing agent for use in PNC applications. However, the catalyst particles remain on the substrate, which is named as 'base-growth'. The strong interaction between the substrate and catalyst leads to a root-growth mechanism.

To further examine the morphology at the nanoscale, TEM images were captured of VACNT addition into the Fe catalyst, which (i) decreased the inner diameter of the CNTs, and (ii) increased the number of walls. 50 different lines were selected for calculation of the inner diameter of the CNTs using the ImageJ software. VACNTs grown from Pure-Fe show a wide distribution in terms of diameter of around $3.27 \mathrm{~nm}$; the Fe15Ni catalyst synthesized VACNTs show a mean diameter of $3.11 \mathrm{~nm}$; and the Fe50Ni catalyst yields mainly small tubes of around $2.52 \mathrm{~nm}$. The TEM images are in clear agreement with the Raman spectroscopy results. The controlled CVD process using $\mathrm{C}_{2} \mathrm{H}_{4}$ as a carbon precursor also helps to achieve smaller diameter CNTs due to its low dissociation probability at high growth temperatures compared to other precursors. However, these TEM results prove that the number of walls of the VACNTs increases in line with the nickel in the catalyst particles, as can be clearly seen in Fig. 7. The number of walls of the VACNTs from Pure-Fe is 2-3 walls, whereas the numbers of walls of the VACNTs synthesized on Fe15Ni and Fe50Ni are in the range of 3-8.

\section{Conclusion}

Herein, the effect that the addition of Ni into an Fe catalyst has on VACNT growth and their many properties, including chirality, diameter, purity, quality, structural morphology and oxidation behavior, was investigated. Through using MA, the addition of $\mathrm{Ni}$ into the Fe catalysts was performed for VACNT growth using CVD. The synthesized VACNTs on different catalysts via CCVD were characterized using Raman spectroscopy, TGA, SEM and TEM. On the basis of the Raman spectroscopy at $532 \mathrm{~nm}$ laser wavelength, the following conclusions were drawn for all of the samples in terms of their peak positions, innermost diameters with metallic or semi conduction branches, Raman mappings, defect length of the VACNTs, and the counts of the common chiralities. To sum up, CNTs with metallic characteristics such as $(9,3),(9,6),(10,4)$ and $(12,3)$ were detected by Raman spectroscopy for all of the samples. The metallic behaviour of the synthesized CNTs with different Fe-Ni catalysts was seen to increase in line with the addition of nickel into the iron. Thus, this method improves the synthesis of VACNTs with metallic behaviour without the use of any etching and treatment methods. Furthermore, the oxidation behavior of the VACNTs was investigated using TGA. We reviewed the control of VACNTs with perfect alignment, as shown in the SEM images of all of the samples. According to the SEM images of VACNTs synthesized on the different catalyst particles, no significant change was observed in their morphologies. However, the VACNTs synthesized on Fe5Ni have a wavier structure compared to others. TEM was used to determine the number of walls and inner diameters of the VACNTs. Further studies on the morphologies of the VACNTs with all of the Fe-Ni catalyst combinations will be carried out. With an increase in the Ni content in the catalysts, the innermost tube diameter of the VACNTs decreased from 3.27 to $2.52 \mathrm{~nm}$ for the Pure-Fe and Fe50Ni catalysts, respectively. These results are consistent with the Raman spectroscopy results. To the best of our knowledge, this is the first study to methodically investigate the relationship between $\mathrm{Fe}-\mathrm{Ni}$ catalyst combinations and the selective growth of VACNTs. The mechanism was explained in terms of how the catalyst combinations affect the chirality distribution of few-walled VACNTs. In a future study, the synthesized different catalysts will be combined with a waterassisted chemical vapor deposition system to enable the growth of VACNTs with a narrow chirality distribution for use in electronic applications.

\section{Conflicts of interest}

There are no conflicts to declare.

\section{Acknowledgements}

This study was financially supported by 38441 Scientific Research Project funded by ITU-Rectorate and TUBITAK 1001 projects (Project No. 112M807 and Project No. 117M290) for the materials and CVD system support. The authors would like to thank Dr H. Enis Karahan, and Zeki Semih Pehlivan for their feedback on the experimental design and discussions. The authors are also grateful to Prof. Haluk Küçük, Assist. Prof. Ali Fuat Ergenç, Assist. Prof. Didem Ovalı, Dr Alptekin Yıldız, Dr Kaan Yıldız, Dr Bihter Zeytuncu, Kıvanç Özışık, Fırat Turgut, Elçin Akar, and Fulden Kaygınok for their support.

\section{References}

1 A. V. Melechko, et al., Vertically aligned carbon nanofibers and related structures: controlled synthesis and directed assembly, J. Appl. Phys., 2005, 97, 1-39.

2 N. M. Briggs and S. P. Crossley, Rapid growth of vertically aligned multi-walled carbon nanotubes on a lamellar support, $R S C A d v .$, 2015, 83945-83952, DOI: 10.1039/c5ra12611h.

3 S. Kar, R. C. Bindal and P. K. Tewari, Carbon nanotube membranes for desalination and water purification: challenges and opportunities, Nano Today, 2012, 7, 385-389.

4 L. Ge, L. Wang, A. Du, M. Hou and Z. Zhu, Vertically-aligned carbon nanotube membranes for hydrogen separation, $R S C$ Adv., 2012, 5329-5336, DOI: 10.1039/c2ra00031h.

5 B. Kim, H. Chung and W. Kim, High-performance supercapacitors based on vertically aligned carbon nanotubes and nonaqueous electrolytes, Nanotechnology, 2012, 23, 1-8.

$6 \mathrm{H}$. K. Mutha, et al., Porosimetry and packing morphology of vertically aligned carbon nanotube arrays via impedance spectroscopy, Nanotechnology, 2017, 28, 1-6. 
7 W. G. Reis, et al., Highly Efficient and Scalable Separation of Semiconducting Carbon Nanotubes via Weak Field Centrifugation, Sci. Rep., 2016, 6, 1-11.

8 R. Zhang, Y. Zhang and F. Wei, Controlled Synthesis of Ultralong Carbon Nanotubes with Perfect Structures and Extraordinary Properties, Acc. Chem. Res., 2017, 50, 179-189.

9 Y. Homma, H. Liu, D. Takagi and Y. Kobayashi, Single-walled carbon nanotube growth with non-iron-group 'catalysts' by chemical vapor deposition, Nano Res., 2009, 2, 793-799.

$10 \mathrm{H}$. Wang, et al., Selective synthesis of single walled carbon nanotubes on metal (iron, nickel or cobalt) sulfate-based catalysts, Carbon, 2018, 129, 128-136.

$11 \mathrm{X}$. Zhao, et al., Selective growth of chirality-enriched semiconducting carbon nanotubes by using bimetallic catalysts from salt precursors, Nanoscale, 2018, 10, 6922-6927.

12 Y. Shen and A. C. Lua, Synthesis of Ni and Ni-Cu supported on carbon nanotubes for hydrogen and carbon production by catalytic decomposition of methane, Appl. Catal., B, 2015, 164, 61-69.

13 G. Chen, et al., Diameter control of single-walled carbon nanotube forests from 1.3-3.0 $\mathrm{nm}$ by arc plasma deposition, Sci. Rep., 2014, 4, 1-7.

14 A. Gohier, C. P. Ewels, T. M. Minea and M. A. Djouadi, Carbon nanotube growth mechanism switches from tip- to base-growth with decreasing catalyst particle size, Carbon, 2008, 46, 1331-1338.

15 S. Esconjauregui, et al., Manipulation of the catalystsupport interactions for inducing nanotube forest growth, J. Appl. Phys., 2011, 109, 1-8.

16 R. Joshi, et al., Catalyst Composition, Morphology and Reaction Pathway in the Growth of 'Super-Long' Carbon Nanotubes, ChemCatChem, 2010, 2, 1069-1073.

17 N. T. Dee, et al., Carbon-assisted catalyst pretreatment enables straightforward synthesis of high-density carbon nanotube forests, Carbon, 2019, 153, 196-205.

18 M. Kumar and Y. Ando, Chemical vapor deposition of carbon nanotubes: a review on growth mechanism and mass production, J. Nanosci. Nanotechnol., 2010, 10, 3739-3758.

19 S. K. Youn, N. Yazdani and H. G. Park, Facile diameter control of vertically aligned, narrow single-walled carbon nanotubes, RSC Adv., 2013, 3, 1434-1441.

20 J. P. Tessonnier and D. S. Su, Recent progress on the growth mechanism of carbon nanotubes: a review, ChemSusChem, 2011, 4, 824-847.

21 V. Jourdain and C. Bichara, Current understanding of the growth of carbon nanotubes in catalytic chemical vapour deposition, Carbon, 2013, 58, 2-39.

22 S. M. Shinde, G. Kalita, S. Sharma and R. Papon, Synthesis of a three dimensional structure of vertically aligned carbon nanotubes and graphene, RSC Adv., 2014, 13355-13360, DOI: 10.1039/c3ra46351f.

23 K. Jiang, C. Feng, K. Liu and S. Fan, A vapor-liquid-solid model for chemical vapor deposition growth of carbon nanotubes, J. Nanosci. Nanotechnol., 2007, 7, 1494-1504.

24 G. D. Nessim, et al., Tuning of vertically-aligned carbon nanotube diameter and areal density through catalyst pretreatment, Nano Lett., 2008, 8, 3587-3593.
25 K. Liu, et al., Controlled termination of the growth of vertically aligned carbon nanotube arrays, Adv. Mater., 2007, 19, 975-978.

26 S. K. Youn and H. G. Park, Morphological evolution of FeMo bimetallic catalysts for diameter and density modulation of vertically aligned carbon nanotubes, J. Phys. Chem. C, 2013, 117, 18657-18665.

27 W. H. Chiang and R. M. Sankaran, Linking catalyst composition to chirality distributions of as-grown single-walled carbon nanotubes by tuning $\mathrm{Ni}_{x} \mathrm{Fe}_{1 x}$ nanoparticles, Nat. Mater., 2009, 8, 882-886.

28 D. Yao, H. Li, Y. Dai and C. Wang, Impact of temperature on the activity of Fe-Ni catalysts for pyrolysis and decomposition processing of plastic waste, Chem. Eng. J., 2020, 408, 1-11, DOI: 10.1016/j.cej.2020.127268.

29 D. Yao and C. Wang, Pyrolysis and in-line catalytic decomposition of polypropylene to carbon nanomaterials and hydrogen over $\mathrm{Fe}$ - and Ni-based catalysts, Appl. Energy, 2020, 265, 114819.

30 M. He, et al., Predominant $(6,5)$ single-walled carbon nanotube growth on a copper-promoted iron catalyst, J. Am. Chem. Soc., 2010, 132, 13994-13996.

$31 \mathrm{~S}$. Li, et al., Wall-number selective growth of vertically aligned carbon nanotubes from FePt catalysts: a comparative study with Fe catalysts, J. Mater. Chem., 2012, 22, 14149-14154.

32 K. Cui, et al., Synthesis of subnanometer-diameter vertically aligned single-walled carbon nanotubes with copperanchored cobalt catalysts, Nanoscale, 2016, 8, 1608-1617.

33 C. M. Seah, S. P. Chai and A. R. Mohamed, Synthesis of aligned carbon nanotubes, Carbon, 2011, 49, 4613-4635.

34 Y. T. Lee, et al., Temperature-dependent growth of carbon nanotubes by pyrolysis of ferrocene and acetylene in the range between 700 and $1000{ }^{\circ} \mathrm{C}$, Chem. Phys. Lett., 2003, 372, 853-859.

35 C. P. Huynh and S. C. Hawkins, Understanding the synthesis of directly spinnable carbon nanotube forests, Carbon, 2010, 48, 1105-1115.

36 S. Zhang, D. Peng, H. Xie, Q. Zheng and Y. Zhang, Investigation on the formation mechanism of double-layer vertically aligned carbon nanotube arrays via single-step chemical vapour deposition, Nano-Micro Lett., 2017, 9, 1-7.

37 K. Hata, et al., Water-assisted highly efficient synthesis of impurity-free single-walled carbon nanotubes, Science, 2004, 306, 1362-1364.

38 P. B. Amama, et al., Role of water in super growth of singlewalled carbon nanotube carpets, Nano Lett., 2009, 9, 44-49.

39 A. W. Orbaek, et al., Single walled carbon nanotube growth and chirality dependence on catalyst composition, Nanoscale, 2013, 5, 9848-9859.

40 M. F. C. Fiawoo, et al., Evidence of correlation between catalyst particles and the single-wall carbon nanotube diameter: a first step towards chirality control, Phys. Rev. Lett., 2012, 108, 1-5.

41 C. P. Deck and K. Vecchio, Prediction of carbon nanotube growth success by the analysis of carbon-catalyst binary phase diagrams, Carbon, 2006, 44, 267-275. 
42 C. Zoican Loebick, D. Abanulo, M. Majewska, G. L. Haller and L. D. Pfefferle, Effect of reaction temperature in the selective synthesis of single wall carbon nanotubes (SWNT) on a bimetallic CoCr-MCM-41 catalyst, Appl. Catal., A, 2010, 374, 213-220.

43 M. V. Kharlamova, et al., Chiral vector and metal catalystdependent growth kinetics of single-wall carbon nanotubes, Carbon, 2018, 133, 283-292.

44 J. A. Elliott, Y. Shibuta, H. Amara, C. Bichara and E. C. Neyts, Atomistic modelling of CVD synthesis of carbon nanotubes and graphene, Nanoscale, 2013, 5, 6662-6676.

45 L. Zheng, G. Sun and Z. Zhan, Tuning array morphology for high-strength carbon-nanotube fibers, Small, 2010, 6, 132-137.

46 S. Esconjauregui, C. M. Whelan and K. Maex, The reasons why metals catalyze the nucleation and growth of carbon nanotubes and other carbon nanomorphologies, Carbon, 2009, 47, 659-669.

47 C. Vets and E. C. Neyts, Stabilities of Bimetallic Nanoparticles for Chirality-Selective Carbon Nanotube Growth and the Effect of Carbon Interstitials, J. Phys. Chem. C, 2017, 121, 15430-15436.

48 M. Aslan, J. B. A. Davis and R. L. Johnston, Global optimization of small bimetallic Pd-Co binary nanoalloy clusters: a genetic algorithm approach at the DFT level, Phys. Chem. Chem. Phys., 2016, 18, 6676-6682.

49 D. Dutta, W. H. Chiang, R. M. Sankaran and V. R. Bhethanabotla, Epitaxial nucleation model for chiralselective growth of single-walled carbon nanotubes on bimetallic catalyst surfaces, Carbon, 2012, 50, 3766-3773.

50 R. Rao, D. Liptak, T. Cherukuri, B. I. Yakobson and B. Maruyama, In situ evidence for chirality-dependent growth rates of individual carbon nanotubes, Nat. Mater., 2012, 11, 213-216.

51 P. R. Rios and A. F. Padilha, Precipitation from Austenite, Encycl. Biomater. Biomed. Eng., 2005, 1-7, DOI: 10.1016/b008-043152-6/01408-x.

52 Y. Kuwayama, K. Hirose, N. Sata and Y. Ohishi, Phase relations of iron and iron-nickel alloys up to $300 \mathrm{GPa}$ implications for composition and structure of the Earth's inner core, Earth Planet. Sci. Lett., 2008, 273, 379-385.

53 J. M. Yan, X. B. Zhang, S. Han, H. Shioyama and Q. Xu, Magnetically recyclable Fe-Ni alloy catalyzed dehydrogenation of ammonia borane in aqueous solution under ambient atmosphere, J. Power Sources, 2009, 194, 478-481.

54 A. Cao, C. Xu, J. Liang, D. Wu and B. Wei, X-ray diffraction characterization on the alignment degree of carbon nanotubes, Chem. Phys. Lett., 2001, 344, 13-17, DOI: 10.1016/ S0009-2614(01)00671-6.

55 P. T. Araujo, et al., Third and fourth optical transitions in semiconducting carbon nanotubes, Phys. Rev. Lett., 2007, 98, 1-4.

56 M. Arefin, Empirical Equation Based Chirality $(n, m)$ Assignment of Semiconducting Single Wall Carbon Nanotubes from Resonant Raman Scattering Data, Nanomaterials, 2012, 3, 1-21.

57 J. R. Sanchez-Valencia, et al., Controlled synthesis of singlechirality carbon nanotubes, Nature, 2014, 512, 61-64.

58 Y. Tian, H. Jiang, P. Laiho and E. I. Kauppinen, Validity of Measuring Metallic and Semiconducting single-Walled
Carbon Nanotube Fractions by Quantitative Raman Spectroscopy, Anal. Chem., 2018, 90, 2517-2525.

59 J. H. Lehman, M. Terrones, E. Mansfield, K. E. Hurst and V. Meunier, Evaluating the characteristics of multiwall carbon nanotubes, Carbon, 2011, 49, 2581-2602.

60 D. Zhang, et al., $(n, m)$ Assignments and quantification for single-walled carbon nanotubes on $\mathrm{SiO}_{2} / \mathrm{Si}$ substrates by resonant Raman spectroscopy, Nanoscale, 2015, 7, 10719-10727.

61 Q. Cheng, S. Debnath, E. Gregan and H. J. Byrne, Vibrational mode assignments for bundled single-wall carbon nanotubes using Raman spectroscopy at different excitation energies, Appl. Phys. A: Mater. Sci. Process., 2011, 102, 309-317.

$62 \mathrm{M}$. Cheng, et al., Selective growth of semiconducting singlewall carbon nanotubes using SiC as a catalyst, Carbon, 2018, 135, 195-201.

63 M. Fouquet, et al., Effect of catalyst pretreatment on chirality-selective growth of single-walled carbon nanotubes, J. Phys. Chem. C, 2014, 118, 5773-5781.

64 L. Zhang, et al., Selective Growth of Metal-Free Metallic and Semiconducting Single-Wall Carbon Nanotubes, Adv. Mater., 2017, 29, 1-9.

65 C. Herrero-Latorre, J. Álvarez-Méndez, J. Barciela-García, S. García-Martín and R. M. Peña-Crecente, Characterization of carbon nanotubes and analytical methods for their determination in environmental and biological samples: a review, Anal. Chim. Acta, 2015, 853, 77-94.

66 A. C. Ferrari, Raman spectroscopy of graphene and graphite: disorder, electron-phonon coupling, doping and nonadiabatic effects, Solid State Commun., 2007, 143, 47-57.

67 D. Ürk, et al., Understanding the polymer type and CNT orientation effect on the dynamic mechanical properties of high volume fraction CNT polymer nanocomposites, Compos. Struct., 2016, 155, 255-262.

68 L. G. Cançado, et al., Quantifying defects in graphene via Raman spectroscopy at different excitation energies, Nano Lett., 2011, 11, 3190-3196.

69 J. E. Proctor, et al., High-pressure Raman spectroscopy of graphene, Phys. Rev. B, 2009, 80, 1-4.

70 K. I. Sasaki, et al., Kohn anomalies in graphene nanoribbons, Phys. Rev. B, 2009, 80, 1-12.

71 L. Qu, F. Du and L. Dai, Preferential syntheses of semiconducting vertically aligned single-walled carbon nanotubes for direct use in FETs, Nano Lett., 2008, 8, 2682-2687.

72 F. Ding, et al., The importance of strong carbon-metal adhesion for catalytic nucleation of single-walled carbon nanotubes, Nano Lett., 2008, 8, 463-468.

73 F. Zhang, et al., Growth of semiconducting single-wall carbon nanotubes with a narrow band-gap distribution, Nat. Commun., 2016, 7, 1-8.

74 M. V. Kharlamova, et al., Chirality-dependent growth of single-wall carbon nanotubes as revealed inside nano-test tubes, Nanoscale, 2017, 9, 7998-8006.

75 C. Li and T. W. Chou, Failure of carbon nanotube/polymer composites and the effect of nanotube waviness, Composites, Part A, 2009, 40, 1580-1586. 